\title{
On the Origin of Low Thermal Conductivity in High Thermoelectric Performance in $n$-type BiAgSeS
}

\author{
Haijun Wu, Y Yan-Ling Pei, Jing Li, Jiehe Sui, Li-Dong Zhao ${ }^{2}$ and Jiaqing $\mathrm{He}^{1,4}$
}

1. Frontier Institute of Science and Technology (FIST), Xi'an Jiaotong University, Xi'an 710054, China

2. School of Materials Science and Engineering, Beihang University, Beijing 100191, China

3. School of Materials Science and Engineering, Harbin Institute of Technology, Harbin 150001, China

4. Department of Physics, South University of Science and Technology of China, Shenzhen 518005, China

Thermoelectric energy conversion technology, which can be used to convert wasted heat into electricity, has received much attention in the past decade. The efficiency of thermoelectric devices is characterized by the dimensionless figure of merit, defined as $Z T=\left(S^{2} \sigma / \kappa\right) T$, where $S, \sigma, \kappa$, and $T$ are the Seebeck coefficient, the electrical conductivity, the thermal conductivity, and the absolute temperature, respectively.[1] Recently, single phase thermoelectric materials with intrinsically low thermal conductivity have been reported, such as $\mathrm{BiCuSeO},[2]$ $\mathrm{Cu}_{3} \mathrm{SbSe}_{4}$,[3] and $\mathrm{CsBi}_{4} \mathrm{Te}_{6}$.[4] Interestingly, all these are $p$-type materials. Surprisingly, single phase $n$-type thermoelectric materials are reported little. Thus, in order to obtain high $Z T$ of a whole thermoelectric device, consisting of p-type and $n$-type thermoelectric elements, $n$-type thermoelectric materials with intrinsically low thermal conductivity are urgent.

In this presentation, we report promising thermoelectric performance in $n$-type polycrystalline BiAgSeS with intrinsically low thermal conductivity ( $\sim 0.29 \mathrm{Wm} \mathrm{K}$ ). Transmission electron microscopy (TEM) results show the micro-features of BiAgSeS including meso-scale grain boundaries, twinned structures, lamellar structures, and nano-scale laminated endotaxial precipitates, which can allow effectively scatter heat-carrying phonons with all length scales.[5] To clarify the possible mechanisms in reducing the latticethermal conductivity of the n-type BiAgSeS, we calculated the lattice thermal conductivity based on our TEM observations using the Callway's model.

In order to further explore the microstructural base of the intrinsically low thermal conductivity of BiAgSeS, the sample was investigated by transmission electron microscopy (TEM).

Fig. 1(a) shows a typical low magnification TEM image with average grain sizes around $80 \mathrm{~nm}$. The twinned structure is clearly shown in Fig. 1(b). Electron diffraction pattern and fast Fourier transforms (FFT) images of two twin variants in Fig. 1(c) confirm $\Sigma 3$ twin along [101] zone axis of BiAgSeS (Fm-3m). Twin boundaries can act as an alternative phonon scattering source while have a neglect effect on electronic transport since the twin boundary is always coherent interfaces, which cannot disrupt the electronic transport with very short wave length.

Fig. 2 shows another micro-feature in BiAgSeS: nano-scale laminated precipitates distributed in the matrix. The inset magnified area shows laminated precipitates $(5-15 \mathrm{~nm})$ distributing disorderly in the matrix. The electron diffraction pattern in Fig. 2(b-d) can be indexed as the coexistence of BiAgSeS cubic $(F m-3 m)$ matrix along [101]C zone axis and $\mathrm{AgBiSe}_{2}$ rhombohedral $(R-3 m)$ precipitates with two variants along [100] $\mathrm{R}$ zone axis. Interestingly, these phases with laminated nanostructure could exist in BiAgSeS with rocksalt cubic structure. This micro-feature could be associated with the phase transitions (hexagonal, rhombohedral and cubic) in $\mathrm{AgBiSe}_{2}$. It is believed that these laminated nano-phases will contribute to the intrinsically low thermal conductivity in BiAgSeS system. The precipitates with two variants can match with the matrix endotaxially, which can achieve concurrent phonon blocking and charge transmitting. High-magnification TEM image in Fig. 2(e) shows nano-scale laminated precipitates with two variants (e.g.. "A" and "B") separated by non-laminated matrix (e.g. "C"). Accordingly, FFT images from these three different areas shown in the right of Fig. 2(e) further confirm the relation between laminated structure with two variants and the non-laminated matrix. 
References:

1. Kanatzidis, M.G., Chemistry of materials, 2009. 22(3): p. 648-659.

2. Li, J., et al., Energy \& Environmental Science, 2012. 5(9): p. 8543-8547.

3. Skoug, E.J. and D.T. Morelli, Physical Review Letters, 2011. 107(23): p. 235901.

4. Chung, D.-Y., et al., Science, 2000. 287(5455): p. 1024-1027.

5. Biswas, K., et al., Nature, 2012. 489(7416): p. 414-8.
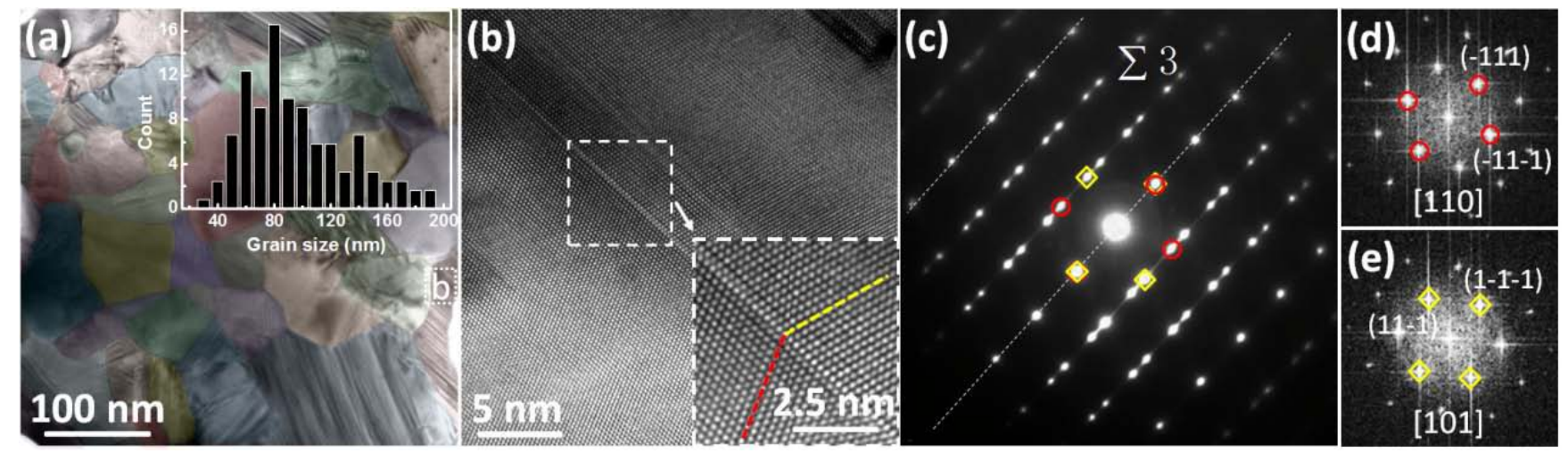

Figure 1. Meso-scale grains and twinned structures of BiAgSeS: (a) Low-magnification TEM image reveals mesoscale grains with inset grain size distribution histogram. (b) HRTEM image shows twinned structure, inset magnified image clearly shows the twin boundary. (c) Electron diffraction pattern of (b). (d) and (e) FFT image of the lower-left and top-right variants in (b) respectively.
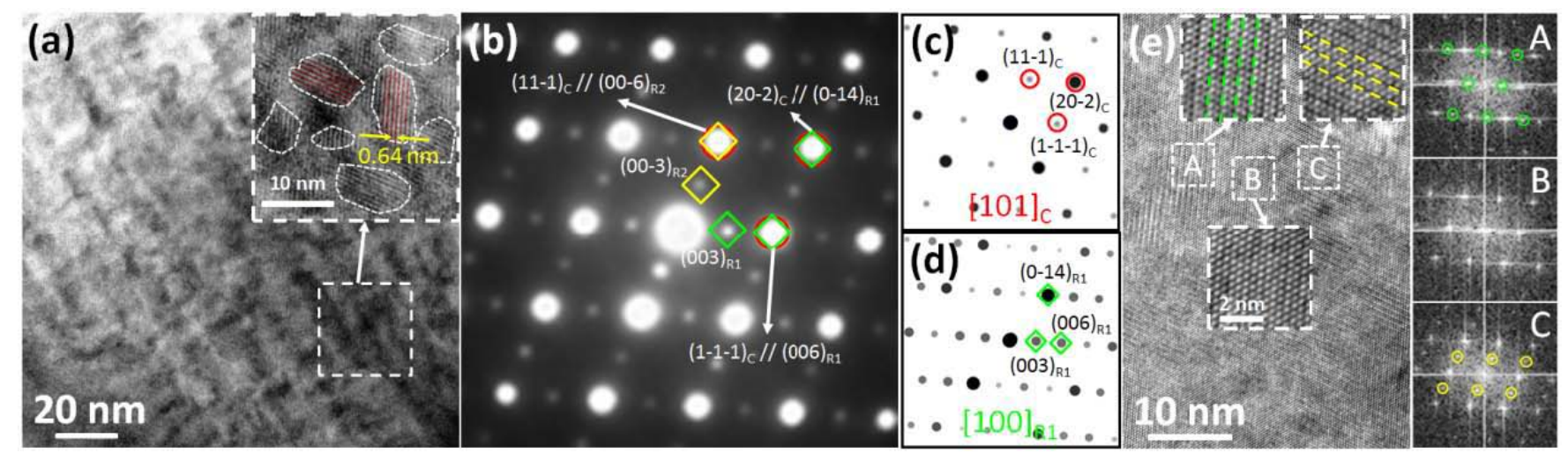

Figure 2. Nano-scale laminated endotaxial precipitates of BiAgSeS: (a) Medium-magnification TEM image shows nano-scale laminated structures, and the inset magnified area shows that laminated precipitates with size range of about 5-15 nm distributes disorderly in the matrix. (b) Electron diffraction pattern of (a). (c) Simulated electron diffraction pattern of BiAgSeS cubic phase along [101] zone axis. (d) Simulated electron diffraction pattern of AgBiSez rhombohedral phase along [100] zone axis. (e) HRTEM image shows nano-scale laminated precipitates with two variants (e.g. "A" and "B") distributing in the non-laminated matrix (e.g. "C"). The responding FFT images of "A", "B" and " $C$ " are showed in the right area. 